\title{
Jogos Sérios em Interação Humano-Computador: Uma revisão Sistemática de Literatura
}

\author{
André Barros de Sales - Universidade de Brasília - UnB - andrebdes@ unb.br Gabriel de \\ Souza Clímaco - Universidade de Brasília - UnB - gabrielsclimaco@ gmail.com Márcia \\ Barros de Sales - Universidade Federal de Santa Catarina - marcia.barros@ufsc.br
}

\begin{abstract}
Resumo - Nos cursos de engenharia de software relacionados, o reconhecimento do ensino de Interação Humano-Computador (IHC) cada vez maior, devido à sua importância para o desenvolvimento de software, e está sendo abordado de muitas maneiras diferentes. Este artigo tem como objetivo apresentar uma revisão sistemática de trabalhos acadêmicos, na publicados na área de engenharia de software, que utilizaram jogos sérios como apoio ao ensino da disciplina de Interação humano-computador, de artigos publicados em base de dados indexadas como IEEE, ACM, Google Acadêmico, entre outros no período de 2007 a 2015. O resultado encontrado apresentou que ainda são incipientes pesquisas neste contexto, e evidenciou a necessidade de realizar uma revisão sistemática em eventos acadêmicos similares, em decorrência da escassez de artigos encontrados em relação a quantidade de artigos publicados na área de engenharia de software e interface humano-computador.
\end{abstract}

Palavras-chaves- Interação Humano-Computador, ensino, revisão sistemática, jogos sérios, engenharia de software.

\section{Serious Games in Human-Computer Interaction: A Systematic Review of Literature}

\begin{abstract}
In the related software engineering courses, recognition of teaching Human-Computer Interaction (HCI) increasing due to its importance for the development of software, and is being addressed in many different ways. This article aims to present a systematic review of academic papers in published in software engineering, who used serious games to support the teaching of the discipline of human-computer interaction, articles published on the basis of indexed data as IEEE, ACM, Google Scholar, and others in the period 2007 to 2015. The results found showed that is still incipient research in this context, and highlighted the need for a systematic review of similar academic events, due to the shortage of items in relation to the quantity articles published in software engineering and human-computer interface.
\end{abstract}

Keywords - Human-Computer Interaction, education, teaching, systematic review, serious games, games, software engineering.

\section{Introdução}

A disciplina de IHC estuda fenômenos que cercam os sistemas computacionais de modo que se tratando de projeto, avaliação e implementação dos mesmos, esses sejam mais interativos para o uso humano. Em outras palavras, IHC tenta melhorar a experiência de pessoas com os computadores, de maneira a torná-la mais produtiva e eficiente e torná-los mais agradáveis ao uso (Rusu \& Rusu 2007).

V. $14 \mathrm{~N}^{\mathrm{o}}$ 1, julho, 2016 
2

Vale ressaltar que a aplicação de IHC é de extrema importância e deve ser evidente e bem entendida na concepção de programas de Ciência da Computação, por parte dos profissionais de software, em todos os níveis (Deng et al. 2009; Pow-Sang 2009).

Por mais que pareça uma boa ideia na teoria, na prática a aplicação de tais conceitos não é tão simples. Existe alguns profissionais da área que, ainda, consideram IHC uma questão secundária (Chewar et al. 2004; Lester 2008). Além disso, o ensino de IHC pode promover uma visão crítica sobre a importância da abordagem do projeto voltado ao usuário, pois tal abordagem permite que os alunos aprendam como desenvolver sistemas que obtêm respostas positivas dos usuários, além de diminuir o custo com a reformulação de um sistema que não inclui quem vai utilizá-lo no processo (Cooke \& Mings 2005).

A disciplina de IHC para os discentes pode lhes fornecer informações cruciais para 0 desenvolvimento de softwares de maior qualidade usáveis e acessíveis, através dos estudos dos fenômenos que envolvem sistemas de computador, design e interação com o usuário, com a intenção de torná-los mais inteligíveis e interativos para o uso humano. O conteúdo da disciplina e sua aplicação apresenta a necessidade de oferecer ao aluno recursos didáticos que o envolva no processo de aprendizagem como (Júnior, 2010; Cerutti, 2010; Sales et al, 2012; Sales et al, 2013).

Tendo em vista a importância do ensino de IHC para o desenvolvimento de softwares, os jogos educativos podem ser uma ferramenta de auxílio nessa aprendizagem. $\mathrm{O}$ uso de jogos educativos para apoiar o processo de ensino e aprendizagem, tem sido considerada uma ferramenta poderosa, em função do seu conteúdo de natureza lúdica e interativa (Sá et al, 2007). Portanto, aliando esses dois fatos, este artigo apresenta uma revisão sistemática da literatura, a fim de encontrar empregos que estão relacionados com ambas as áreas do conhecimento

Os jogos digitais educacionais ao longo dos anos ganharam interfaces gráficas mais interativas, desde então houve um extremo crescimento nessa área, sendo que a grande maioria de tudo o que já foi desenvolvido possui caráter predominantemente lúdico. Porém existe dentro da área de jogos, uma classificação denominada como: jogos sérios. Jogos sérios (serious games) podem ser classificados como aqueles cujos objetivos vão além do entretenimento (Ye et al. 2007). Cabe dizer então que nesse contexto, a palavra "sério" faz referência ao propósito do jogo e não ao seu conteúdo (Conte et al. 2014).

É possível encontrar vários trabalhos dentro da área de jogos sérios passíveis de aplicação em conteúdos relacionados à engenharia de software, como por exemplo: programação, gerenciamento de projeto, fundamentos da computação. Mesmo com o amplo uso de jogos para o ensino de computação, não é comum que se desenvolva algo dentro da área de Interação Humano-computador.

Esse artigo está organizado em cinco seções. Está seção contextualizou o trabalho, a segunda seção apresenta uma breve explicação sobre revisão sistemática e o método utilizado. A seção três apresentação dos resultados e discussão. A seção quatro tece algumas considerações finais.

\section{A Revisão Sistemática}

A revisão sistemática é uma metodologia de estudo secundário que visa estabelecer um levantamento formal do estado da arte de forma robusta e consistente, a partir de um planejamento e execução criteriosos (Biolchini et al. 2005). As revisões sistemáticas são planejadas para serem metódicas, explícitas e passíveis de reprodução (Sampaio e Mancini 2007). De modo que o processo seja conduzido segundo uma sequência metodologicamente bem definida de etapas, de acordo com um protocolo previamente planejado (Munzlinger et al. 2012).

A revisão sistemática foi realizada em três etapas: planejamento, execução da pesquisa e análise dos resultados obtidos, descritas nas próximas subseções. 


\subsection{Planejamento}

\subsubsection{Definição das questões de pesquisa}

- Q1 - Quantos jogos sérios atualmente podem ser aplicados na disciplina de Interação HumanoComputador?

- Q2 - Quais são os jogos referentes à questão Q1?

- Q3 - Qual dos jogos referentes à Q1 é o melhor a ser aplicado dentro da disciplina de IHC?

\subsubsection{Seleção de fontes}

A string de busca foi aplicada na base de dados http://www.periodicos.capes.gov.br/, pois a mesma qual engloba artigos publicados em outras bases de dados, tais como IEEE, ACM, entre outros, e artigos publicados em eventos.

\subsubsection{Idiomas}

Foram considerados artigos publicados tanto em inglês como em português.

\subsubsection{String de Busca}

População: Engenharia de Software

P: (Software Development <or> Software Engineering)

Intervenção: Jogos sérios educativos

I: (serious games <or>educational games)

Outcome: forma como os benefícios serão encontrados

O: (human interaction computer <or> usability)

String de busca final: $\mathrm{P}<$ and $>\mathrm{I}<\mathrm{and}>\mathrm{O}$

String de Busca: ((Software Development <or> Software Engineering) <and> (serious games <or> educational games) <and> (human computer interaction <and> usability <or> teaching))

\subsubsection{Restrições}

- Critérios de inclusão:

Os artigos incluídos na pesquisa cumpriam os seguintes critérios:

- I1 - Os artigos devem estar disponíveis na web;

o I2 - Os artigos devem tratar do tema de jogos sérios;

o I3 - Os artigos devem ser da área de engenharia de software.

- Critérios de exclusão:

Foram excluídos artigos que se enquadravam em algum dos critérios:

- E1 - Tratavam de jogos sérios, mas não dentro da área de engenharia de software;

o $\quad$ E2 - Artigos que são da área de engenharia de software, porém não de algum assunto que não Interação Humano - Computador.

\subsubsection{Periodo de busca}

Foram considerados artigos publicados somente no período entre 2007 e 2015.

\section{Resultados e Discussão}

Nos resultados obtidos nas bases indexadas, foram encontrados um total de 100 artigos que tratavam sobre o assunto de jogos sérios. Desses somente 27 artigos tratavam do desenvolvimento de um jogo de outras áreas do conhecimento. Exclusivamente outros 14 artigos abordavam sobre jogos ou games para a área de engenharia de software.

O quadro 1 a seguir, apresenta uma visão dos 14 artigos, que foram encontrados com a string: "jogos ou games" + "área de Engenharia de Software" publicados em 2007 a 2015, ordenados por um 
número seqüencial, título do artigos, o endereço do artigo na internet, os autores, instituição dos autores e o ano de publicação.

Quadro 1 - Artigos classificados pelos critérios de inclusão I2 e I3

\begin{tabular}{|c|c|c|c|c|c|}
\hline ID & Título & Endereço & Autores & $\begin{array}{l}\text { Institui } \\
\text { ção }\end{array}$ & Ano \\
\hline A1 & $\begin{array}{l}\text { Um Jogo para o Ensino de Engenharia } \\
\text { de Software Centrado na Perspectiva } \\
\text { de Evolução }\end{array}$ & $\begin{array}{l}\text { http://migre. } \\
\text { me/uibJS }\end{array}$ & \begin{tabular}{llr|} 
Eduardo & Figueiredo, Cidiane Lobato, \\
Klessis Dias, Julio Leite, Carlos \\
Lucena
\end{tabular} & $\begin{array}{l}\text { PUC- } \\
\text { Rio }\end{array}$ & 2007 \\
\hline A2 & $\begin{array}{l}\text { SimulES-W: Um Jogo para o Ensino } \\
\text { de Engenharia de Software }\end{array}$ & $\begin{array}{l}\text { http://migre. } \\
\text { me/uiecv }\end{array}$ & $\begin{array}{l}\text { Elizabeth Suescún Monsalve, Vera } \\
\text { M. B. Werneck, Julio C. S. do Prado } \\
\text { Leite. }\end{array}$ & $\begin{array}{l}\text { PUC- } \\
\text { Rio }\end{array}$ & 2010 \\
\hline A3 & $\begin{array}{l}\text { Sobre o uso de Jogos Digitais para o } \\
\text { Ensino de Engenharia de Software }\end{array}$ & $\begin{array}{l}\text { http://migre. } \\
\text { me/uiedc }\end{array}$ & $\begin{array}{l}\text { Lúcia Fernandes e Cláudia M. Lima } \\
\text { Werner }\end{array}$ & $\begin{array}{l}\text { COPPE/ } \\
\text { UFRJ }\end{array}$ & 2009 \\
\hline A4 & $\begin{array}{l}\text { Promovendo a Aprendizagem de } \\
\text { Engenharia de Requisitos de Software } \\
\text { Através de um Jogo Educativo }\end{array}$ & $\begin{array}{l}\text { http://migre. } \\
\text { me/uiefr }\end{array}$ & $\begin{array}{l}\text { Marcello Thiry, Alessandra Zoucas, } \\
\text { Rafael Q. Gonçalves }\end{array}$ & Univali & 2010 \\
\hline A5 & $\begin{array}{lcr}\text { Um Modelo de Avaliação de Jogos } \\
\text { Educacionais } \\
\text { Software }\end{array}$ & $\begin{array}{l}\text { http://migre. } \\
\text { me/uiehH }\end{array}$ & $\begin{array}{lcc}\text { Rafael Savi; } & \text { Christiane } & \text { G. } \\
\text { Wangenheim, Adriano F.Borgatto }\end{array}$ & UFSC & 2011 \\
\hline A6 & 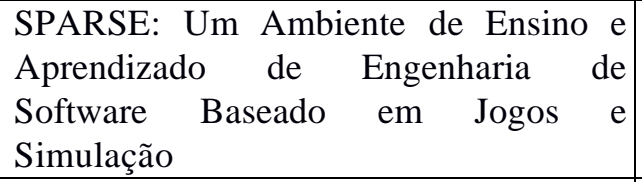 & $\begin{array}{l}\text { http://migre. } \\
\text { me/uiek7 }\end{array}$ & $\begin{array}{l}\text { Mariane M. Souza, Rodolfo F. } \\
\text { Resende, Lucas S. Prado , Edgar F. } \\
\text { Fonseca, Flavio A. Carvalho, } \\
\text { Alexsander D. Rodrigues }\end{array}$ & UFMG & 2010 \\
\hline A7 & $\begin{array}{l}\text { InspectorX: Um Jogo para o } \\
\text { Aprendizado em Inspeção de Software }\end{array}$ & $\begin{array}{l}\text { http://migre. } \\
\text { me/uiemE }\end{array}$ & Henrique Pötter, Marcelo Schots & UFRJ & 2011 \\
\hline A8 & $\begin{array}{l}\text { iTest Learning: Um Jogo para o } \\
\text { Ensino do Planejamento de Testes de } \\
\text { Software }\end{array}$ & $\begin{array}{l}\text { http://migre. } \\
\text { me/uienB }\end{array}$ & \begin{tabular}{llr|} 
Virgínia & Farias, Carla Moreira, \\
Emanuel & Coutinho, Ismayle & S. \\
Santos. & &
\end{tabular} & UFC & 2012 \\
\hline A9 & $\begin{array}{l}\text { Avaliação da Aprendizagem em } \\
\text { Experimentos com Jogo Educativo de } \\
\text { Engenharia de Requisitos }\end{array}$ & $\begin{array}{l}\text { http://migre. } \\
\text { me/uieoG }\end{array}$ & $\begin{array}{l}\text { Rafael Q. Gonçalves, Marcello Thiry } \\
\text { e Alessandra Zoucas }\end{array}$ & Univali & 2011 \\
\hline A10 & $\begin{array}{l}\text { UsabilityGame: jogo simulador para } \\
\text { apoio ao ensino de usabilidade. }\end{array}$ & $\begin{array}{l}\text { http://migre. } \\
\text { me/uier6 }\end{array}$ & $\begin{array}{llll}\begin{array}{l}\text { Sommariva, } \\
\text { Vavassori, D.: }\end{array} & \text { L., } & \text { Benitti, } & \text { F., } \\
\end{array}$ & Univali & 2011 \\
\hline A11 & $\begin{array}{l}\text { Um Jogo de Estratégia de Gerência de } \\
\text { Configuração }\end{array}$ & $\begin{array}{l}\text { http://migre. } \\
\text { me/uietp }\end{array}$ & $\begin{array}{l}\text { Karen Figueiredo, Jonatas Ferreira, } \\
\text { Leonardo Murta, Esteban Clua }\end{array}$ & UFF & 2010 \\
\hline A12 & $\begin{array}{l}\text { Análise de Jogos Educativos de } \\
\text { Computador para Gerência de Projetos } \\
\text { de Software }\end{array}$ & $\begin{array}{l}\text { http://migre. } \\
\text { me/uieve }\end{array}$ & Lauriana Paludo, André Raabe & Univali & 2010 \\
\hline A13 & $\begin{array}{l}\text { Panorama do Ensino de Engenharia de } \\
\text { Software em Cursos de Graduação } \\
\text { Focado em Teste de Software: Uma } \\
\text { Proposta de Aprendizagem Baseada } \\
\text { em Jogos }\end{array}$ & $\begin{array}{l}\text { http://migre. } \\
\text { me/uiexA }\end{array}$ & $\begin{array}{l}\text { Tarcila Gesteira da Silva, Felipe M. } \\
\text { Müller, Giliane Bernardi }\end{array}$ & UFSM & 2011 \\
\hline A14 & $\begin{array}{l}\text { UsabiliCity: Um Jogo de Apoio ao } \\
\text { Ensino de Propriedades de } \\
\text { Usabilidade de Software Através de } \\
\text { Analogias. }\end{array}$ & $\begin{array}{l}\text { http://migr } \\
\text { e.me/uiez8 }\end{array}$ & \begin{tabular}{lll|} 
Bruna M. Ferreira, Luis & Rivero, \\
Adriana Lopes, Anna & Beatriz \\
Marques, Tayana Conte &
\end{tabular} & UFAM & 2014 \\
\hline
\end{tabular}

O quadro 2 mostra os dois trabalhos selecionados. A primeira coluna do quadro contém uma numeração sequencial para os trabalhos; a segunda apresenta os títulos dos trabalhos que foram lidos na 
5

íntegra; a terceira mostra os autores dos trabalhos; a quarta coluna a fonte de pesquisa e última coluna mostra quais critérios foram usados para incluir os trabalhos.

Quadro 2 - Artigos classificados para essa pesquisa

\begin{tabular}{|l|l|l|l|l|}
\hline Id & Título do trabalho & Autores & Fontes & $\begin{array}{l}\text { Critérios de } \\
\text { Inclusão }\end{array}$ \\
\hline 1 & $\begin{array}{l}\text { UsabilityGame: Jogo Simulador para } \\
\text { Apoio ao Ensino de Usabilidade }\end{array}$ & $\begin{array}{l}\text { Sommariva, L., Benitti, F., ACM } \\
\text { Vavassori, D. }\end{array}$ & I1/I2/I3 \\
\hline 2 & $\begin{array}{l}\text { UsabiliCity: Um Jogo de Apoio ao } \\
\text { Ensino de Propriedades de Usabilidade } \\
\text { de Software Através de Analogias }\end{array}$ & $\begin{array}{l}\text { B. M. Ferreira, L. Rivero, A. } \\
\text { Lopes, A. B. Marques, T. Conte }\end{array}$ & $\begin{array}{l}\text { Research } \\
\text { Gate }\end{array}$ & I1/I2/I3 \\
\hline
\end{tabular}

Com relação à questão de pesquisa Q1 é possível afirmar que, atualmente, há apenas 2 jogos que podem ser aplicados dentro dos temas abordados pela disciplina de IHC. Vale ressaltar a observação de que ambos os jogos tratam do tema de usabilidade. Nesse sentido, é possível dizer que o desenvolvimento de jogos na área tratada nesse artigo, tratam do mesmo tema.

Já com relação à Q2, os jogos encontrados referentes à resposta de Q1, foram o UsabilityGame e o Usabilicity. O UsabilityGame é um jogo que simula uma empresa de software em busca de um engenheiro de usabilidade. O conteúdo abordado na aplicação gira em torno do ciclo de vida de engenharia de usabilidade proposto por Mayhew (1999). O jogo possui três fases, cada qual referente a uma etapa do ciclo de vida referenciado acima. Além das 3 fases, a aplicação também conta com 3 ambientes de apoio ao usuário. O primeiro ambiente consiste na "Sala do consultor Nielsen": um ambiente caricaturado no qual o aluno pode consultar informações sobre as heurísticas de usabilidade quando sentir a necessidade.

O segundo ambiente é a "Sala do Engenheiro de Usabilidade Líder": que é um ambiente para guiar o usuário pelos próximos passos caso o mesmo se sinta perdido. Por último, há também a "Sala do Chefe": na qual o jogador pode visualizar informações sobre o seu desempenho em cada etapa do jogo, indicando as etapas que deverão ser mais bem desempenhadas. Há também na aplicação uma interação com o professor, que simula o papel do engenheiro de usabilidade líder.

No artigo encontrado, a avaliação do jogo não chegou a ser executada, apenas planejada. O planejamento envolve duas etapas de avaliação: (i) avaliação do jogo por um especialista (professores da área de IHC) através do instrumento de avaliação definido por (Aguiar 2010); e (ii) avaliação empírica (qualitativa e quantitativa) realizada em uma turma de IHC.

O Usabilicity, por outro lado, trata da simulação de uma cidade que apresenta gargalos estruturais, e a finalidade do jogador é aplicar os conceitos de usabilidade para que seus problemas possam ser resolvidos. O conteúdo trabalhado em específico são as propriedades de usabilidade descritas na técnica de Avaliação Heurística (AH) propostas por Nielsen (1994).

A aplicação é constituída por 5 fases. Em cada fase são apresentados três elementos. O primeiro elemento é constituído pela indicação do número da fase e o seu título com relação ao problema de usabilidade. O segundo elemento apresenta a descrição para os problemas a serem resolvidos na fase, sendo que em cada fase são apresentados dois problemas distintos entre si e distintos dos problemas das demais fases, e para cada problema, o jogador deverá selecionar uma heurística correspondente para propor uma solução. Por fim, o terceiro elemento apresenta as heurísticas para seleção do jogador. Com o intuito de chamar a atenção do jogador, cada heurística é representada por um personagem.

Assim como o UsabilityGame, o Usabilicity apresenta uma tela de apoio ao usuário. No caso desta aplicação a tela de apoio é apresentada logo após a tela inicial, e apresenta informações sobre as heurísticas de usabilidade.

A avaliação do jogo, assim como no planejado por (Sommariva et al. 2011), é constituído de duas etapas: (i) avaliação feita por uma turma de ciência da computação, a qual foi aplicado o jogo, por meio de 
um modelo de questionário desenvolvido por (Lang 1980); e (ii) avaliação feita por professores da área, por meio da aplicação questionário de requisitos de usabilidade e pedagógicos sugerido por (An et al. 2013). Por meio dessa avaliação em questão houve um retorno positivo sobre a qualidade da aplicação.

Para responder Q3 é necessário antes fazer uma observação: o nível de complexidade do segundo trabalho é menor do que o primeiro, visto que trata de um conteúdo mais simples, ainda que dentro do mesmo assunto. Portanto, não há como definir qual das aplicações é melhor para ser aplicada, mas sim, qual delas se encaixa melhor no contexto em que se deseja aplicar.

O UsabilityGame, por ser mais complexo, é aplicável em um contexto onde deseja abordar uma quantidade maior de conteúdo ou o contudo trabalhado pelo jogo em específico. Deve-se tomar também o cuidado de o aplicar em um ambiente em que se tenha mais tempo disponível para uso da aplicação.

O Usabilicity deve ser aplicado em situações que o desejo é abordar o conteúdo de usabilidade de maneira mais rápida e prática, ou conteúdo do próprio jogo em específico. Vale frisar que essa aplicação toma menos tempo para ser aplicada, em vista do fato de ser mais simples.

Por serem aplicações web, ambos os trabalhos dependem de localidade com conexão a internet para serem aplicadas. Ambos os trabalhos foram desenvolvidos para ambiente web. O UsabilityGame foi desenvolvido em PHP enquanto o Usabilicity foi desenvolvido em JavaScript e as artes do jogo foram desenvolvidas na aplicação CorelDraw. O jogos podem ser acessados através dos links:

- UsabilityGame: http://usabilitygame.com/

- Usabilicity: http://usabilicity.appspot.com/

\section{Considerações Finais}

Diversas pesquisas propõem, com sucesso, o uso de jogos para o ensino de computação, e levando em consideração a importância que tem o ensino de IHC para a área da computação, este artigo apresentou uma revisão sistemática com o objetivo de encontrar jogos para apoiar o ensino na área de IHC.

A partir dos resultados obtidos com a revisão sistemática foi possível levantar os trabalhos passíveis de aplicação para o auxílio do ensino de IHC.

Com base no que foi apresentado cabe dizer que há pouco interesse no desenvolvimento de jogos na área de IHC, o que ratifica o fato de que muitos profissionais da área consideram a disciplina como algo secundário, tendo em vista o número de trabalhos encontrados dentro da área de engenharia de software no tema de jogos sérios. Vale também acrescentar que ambos os jogos tratam do tema de usabilidade, que é apenas um dos conceitos em IHC; ou seja, ainda há uma área vasta do conhecimento a ser explorada dentro do conteúdo de Interação Humano-Computador.

Portanto, os resultados obtidos nessa revisão, considerando-se os objetivos e as questões de pesquisa propostas, servem de base para afirmar que faltam jogos dentro da disciplina de IHC, no sentido de apoio ao ensino. Propõe-se como trabalhos futuros o desenvolvimento de jogos para suprir a necessidade de apoio ao ensino da referida disciplina, levando em conta a importância que ela tem para o desenvolvimento de software em geral.

\section{Referencias Bibliográficas}

ACM. Computing Curricula Computer Science. [S.1.]. Disponível em <http://www.acm.org/education/education/education/curric_vols/cc2001.pdf >, 2001.

AGUIAR, M. P. Jogos eletrônicos educativos: instrumento de avaliação focado nas fases iniciais do processo de design. UFPR. Curitiba, 2010. Dissertação de Mestrado em Design.

ALDERSON, P., GREEN, S., HIGGINS, J. P. T. Cochrane Reviewers' Handbook 4.2.2. The Cochrane 
AN, D., DA SIlVA, C., RIBEIRO, D., DA ROCHA, P., MALTINTI, C., NUNES, V., FÁVERO, R. Digita-um Jogo Educativo de Apoio ao Processo de Alfabetização Infantil. Simpósio Brasileiro de Informática na Educação, 2013.

BENITTI, F. B. V. Software Engineering - Role-Playing Game: an interactive game for Software Engineering education. International Journal of Advanced Research in Computer Science, v. 2, n. 2, 2011.

BIOLCHINI, J. C., MIAN, P. G., NATALI, A. C. C., TRAVASSOS, G. H. Systematic review in software engineering. Technical Report ES 679/05, Federal University of Rio de Janeiro, System Engineering and Computer Science Departament, Federal University of Rio de Janeiro, BR, 2005.

CERUTTI, D. M. L. Ensino de IHC: Desconstruindo interfaces em sala de aula. WEIHC - Workshop sobre Ensino de IHC. Belo Horizonte, MG, 2010.

CHEIRAN, J. F. P., MOURA, E. J. R., MELO, A. M. Diretrizes de Acessibilidade para Jogos em Dispositivos Móveis. Revista Jr de Iniciação Científica em Ciências Exatas e Engenharia - ICCEEg, n. 11, p 33-42, 2015.

CHEWAR, C. M., MCCRICKARD, D. S., SOMERVELL, J. Making a Case for HCI: Comparing Materials for Case-Based Teaching. 34th ASEE/IEEE Frontiers in Education Conference, Savannah, 2004.

CONTE, T., FERREIRA, B. M., LOPES, A., MARQUES, A. B., RIVERO, L. UsabiliCity: Um Jogo de Apoio ao Ensino de Propriedades de Usabilidade de Software Através de Analogias. Simpósio Brasileiro de Informática na Educação, p. 1273-1282, 2014.

COOKE, L., MINGS, S. Connecting usability education and research with industry needs and practices. IEEE Transactions on Professional Communication, 48(3), p. 296-312, 2005.

DENG, X., LIANG, L., WANG, Y. Usability Evaluation Driven by Cooperative Software Description Framework. International Joint Conference on Computational Sciences and Optimization, China, p. 364-366, 2009.

HAINEY, T., et al. Evaluation of a game to teach requirements collection and analysis in software engineering at tertiary education level. Computers \& Education, v.56, Issue 1, p. 21-35, 2011.

HILL, J. M. D., et al. Puzzles and games: addressing different learning styles in teaching operating systems concepts, in Proceedings Technical Symposium on Computer Science Education New York, 2003.

JAHRE, M., NATVIG, L., SINDRE, G. Experimental validation of the learning effect for a pedagogical game on computer fundamentals. IEEE Transactions on Education, v. 52, Issue 1, 2009.

JUNIOR, P. T. A. Papéis do Docente em IHC: do conhecimento ao mercado. Ihc 2010 IX Simpósio sobre Fatores Humanos em Sistemas Computacionais. Belo Horizonte, MG. Anais Extendidos do IX Simpósio de Fatores Humanos em Sistemas Computacionais (Volume II). Porto Alegre - RS: Sociedade Brasileira de Computação SBC, 2010. v. 01. p. 79-82.

KHAN, K. S., TERRIET, G., GLANVILLE, J., SOWDEN, A. J., KLEIJNEN, J. Undertaking systematic review of research on effectiveness. Crd's guidance for those carrying out or commissioning reviews. CRD Report Number 4 (2nd Edition), University of York, NHS Centre for Reviews and Dissemination, University of York, USA, 2001.

KOCHANSKI, D., SAVI, R., VON WANGENHEIM, C. G. Revisão Sistemática sobre Avaliação de Jogos Voltados para Aprendizagem de Engenharia de Software no Brasil. Fórum de Educação em Engenharia de Software, Campinas, São Paulo, 2009. 
LESTER, C. Y. Advancing the Multidisciplinary Nature of Human Computer Interaction in a Newly Developed Undergraduate Course. Second International Conferences on Advances in ComputerHuman Interactions, Sainte Luce, p. 177-182, 2008.

LANG, P. Behavioral treatment and bio-behavioral assessment: computer applications. Technology in mental health care delivery systems, Norwood, NJ, Ablex, 1980.

MAYHEW, D. J. The Usability Engineering Lifecycle.San Diego, Academic Press, 1999. MUNZLINGER, E., NARCIZO, F. B., QUEIROZ, J. E. R. Sistematização de revisões bibliográficas em pesquisas da área de IHC. Livro dos Tutoriais do XI Simpósio Brasileiro sobre Fatores Humanos em Sistemas Computacionais, Cuiabá, MT, 2012.

MURATET, M., et al. Experimental Feedback on Prog\&Play: a serious game for programming practice. Computer Graphic Forum, v. 30, Issue 1, p. 61-73, 2011

NAVARRO, E. SimSE: A Software Engineering Simulation Environment for Software Process Education. University of California, 2006. Doctoral Dissertation.

NIELSEN, J. Usability engineering. Morgan Kaufmann Pub, 1994.

PAI, M., MCCULLOCH, M., COLFORD, J. Systematic review: A road map version 2.2. Guidelines 2.2, University of California, Systematic Reviews Group, University of California, USA, 2002.

POW-SANG, J. A. et al. Applying the Chilean Educational Experience in HCI to Peruvian Undergraduate and Graduate Programs. International Conferences on Advances, ComputerHuman Interactions, Cancun, p. 360-364, 2009.

RUSU, C., RUSU, V. Teaching HCI: a challenging intercultural, interdisciplinary, cross-field experience. 1st International conference on intercultural collaboration, Tokyo, p. 344-354, 2007.

SALES, A. B. DE; COELHO, L. D. N. ; SALES, M. B., O Uso de Mapas Conceituais na Disciplina Interação Humano-Computador. Revista Extensio, (Florianópolis), v. 9, p. 66-72, 2012.

SALES, A. B. DE; Del, A. DE M.; SALES, M. B., Avaliação da Aplicação da Aprendizagem Baseada em Problemas na Disciplina de Interação Humano e Computador de curso de Engenharia de Software. RENOTE. Revista Novas Tecnologias na Educação, v.11, n.3, 2013.

SAMPAIO, R. F., MANCINI, M. C. Estudos de revisão sistemática: um guia para síntese criteriosa da evidência científica. Revista Brasileira de Fisioterapia, v. 11, p. 83-89, 2007.

SÁ, E.J.V; TEIXEIRA, J.S.F; FERNANDES, C.T Design de atividades de aprendizagem que usam Jogos como princípio para Cooperação. In: Anais do XVIII Simpósio Brasileiro de Informática na Educação (SBIE), São Paulo - SP, Brasil, 2007.

SMITH, R., GOTEL, O. Gameplay to introduce and reinforce requirements engineering practices, IEEE International Requirements Engineering Conference, Barcelona, 2008.

SOMMARIVA, L., BENITTI, F. B. V., DALCIN, F. S. UsabilityGame: Jogo Simulador para Apoio ao Ensino de Usabilidade. X Simpósio Brasileiro de Fatores Humanos em Sistemas Computacionais, Porto de Galinhas, Pernambuco, Brasil, 2011.

VARGAS, J., GARCÍA-MUNDO, L., GENERO, M., PIATTINI, M.A systematic mapping study on serious game quality. 18th International Conference on Evaluation and Assessment in Software Engineering, 2014.

YE, E., LIU, C., POLACK-WAHL, J. Enhancing Software Engineering Education Using Teaching Aids in 3-D Online Virtual Worlds. ASEE/IEEE 37th Frontiers.Education Conference, Milwaukee WI, 2007. 